\title{
Design of a Reflecting Luneburg Lens by Metal-Only Metasurface
}

\author{
$\underline{\text { J. Ruiz-García }}^{1}$, E. Martini ${ }^{2}$, C. Della Giovampaola ${ }^{3}$, R. Sauleau ${ }^{1}$, D. González-Ovejero ${ }^{1}$, and S. Maci ${ }^{2}$. \\ ${ }^{1}$ Univ. Rennes, CNRS, IETR (Institut d'Électronique et de Télécommunications de Rennes) - UMR 6164, \\ F-35000, Rennes, France. \\ ${ }^{2}$ Department of Information Engineering and Mathematics, University of Siena, Siena 53100, Italy. \\ ${ }^{3}$ Wave Up, Siena 53100, Italy. \\ jorge.ruiz@univ-rennes1.fr
}

\begin{abstract}
This paper presents the implementation of a novel surface-wave (SW) beam-forming lens. The theory of Flat Optics is used in a double-layer parallel plate waveguide (PPW) structure to create in the top PPW a plane wave steerable in the entire azimuth range. By using metallic pins, we have implemented in the bottom PPW a radially-dependent refraction index that, combined with a cylindrical reflector, results in a flat and symmetric system ideal for the excitation of reconfigurable antennas.
\end{abstract}

\section{INTRODUCTION}

Flat Optics (FO) [1] has been recently extended for the development of new wave-guiding devices. FO theory adapts geometrical optics (GO) [2] to surface wave (SW) structures, where the spatial variability of the impedance boundary conditions (IBCs) is exploited to control the SW wavefront. FO principles have been efficiently employed in the implementation of SW lenses [3-5] based on metasurfaces (MTSs). For this kind of lenses, the modulation of the IBCs allows one to manipulate an equivalent refractive index $n_{e q}$, which has the same function as the refractive index in GO. Thus, the FO ray-tracing mechanism for varying IBCs relies on an eikonal equation, as the GO analysis of graded index materials. As for the implementation of these lenses by MTSs, they are made of an arrangement of sub-wavelength elements printed on a grounded dielectric slab [6] or embedded in a metal base-plate [7,8]. Since the MTS constitutive elements are electrically small, when a SW propagates on the MTS the latter can be accurately described by homogenized IBCs. The modification of the MTS geometrical features (e.g., size and orientation) emulates the IBC variation, and, in the case of SW lenses, enables the implementation of $n_{e q}$. For some antenna systems, like the ones based on quasi-optical beam-formers, a guided plane wave is used to illuminate the radiating aperture $[9,10]$. Moreover, the change of the plane wave angle of incidence on this radiating aperture implies the change of the beam direction. However, most feeding designs present limitations in the range of variability of the plane wave incidence angle. Conventional planar Luneburg lenses [11] (when setting several ports, they cannot be in opposite positions) or quasi-optical pillbox systems [12] (horn shifting is restricted by the parabolic reflector attributes and degrades the final radiated beam) are examples of beam-formers limited in azimuthal scanning. Sometimes, this constraint is overcome by setting additional feeding configurations or duplicating the excitation scheme [10]. Here, we present a low profile lens able to provide a plane wave that can follow any azimuthal direction in a circular area where the radiating part of the system will be placed. The proposed architecture includes two stacked parallel plate waveguides (PPWs), a corner reflector, and a waveguide feed. In the lower PPW, the waveguide feed will launch a cylindrical wave. Then, the rays will be directed by the imposed equivalent refractive index to reach the lens border with a given angle. Finally, the corner-reflector will couple the two layers and return a plane wave in the upper layer. While in a conventional Luneburg lens the generated plane wave travels outwards, the presented structure creates a plane wave impinging into the same area occupied by the lens in the bottom PPW. For this reason, we call it "Reflecting Luneburg lens".

\section{Design And Implementation of A Reflecting Luneburg LEnS}

The design problem lies in finding the equivalent refractive index, $n_{e q}$, required to generate a plane wave in the top layer. As indicated in Section I, we must find a distribution of $n_{e q}$ such that the reflected rays form a plane 


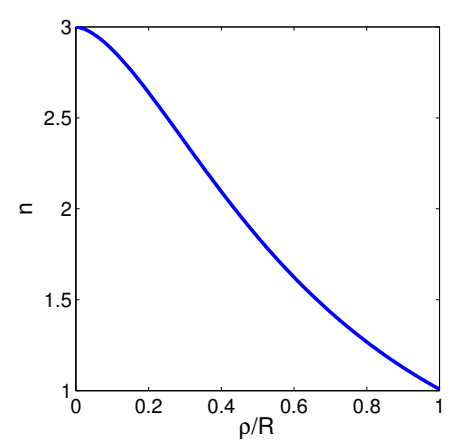

(a) $n(\rho)$

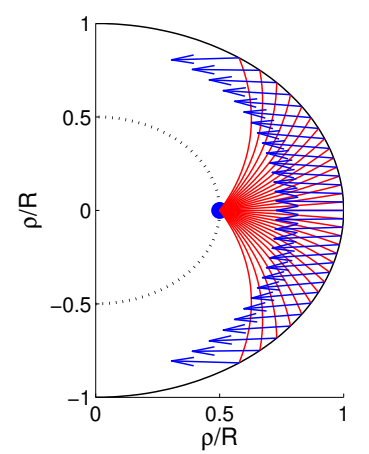

(b) Ray-paths and reflected rays

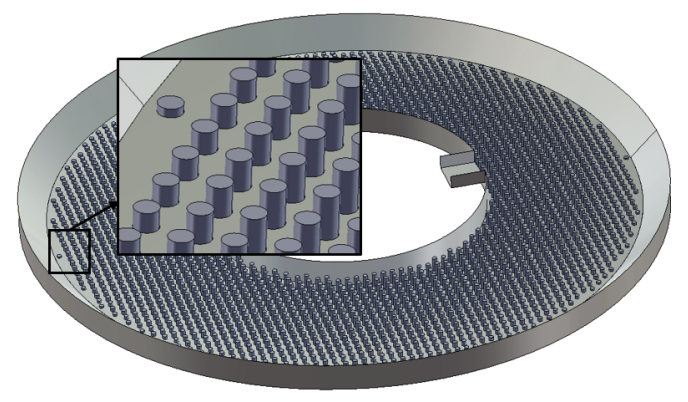

(c) Flat lens by metallic pins

Fig. 1: (a) Optimized equivalent refractive index. (b) Resulting ray-paths (red) and reflected ray directions (blue). (c) MTS lens implemented by varying height cylindrical pins.

wave. To this end, we analyze the direction of the reflected rays by using the ray-tracing technique [1]. We use a cylindrical coordinate system $(\rho, \phi, z)$ for the structure description. The process essentially consists in optimizing a radial distribution of the refractive index $n_{e q}(\rho)$ so as to obtain parallel reflected rays along a section of the lens perimeter illuminated by the source. This circumferential region is at the rim of the lens and in front of the source position. For the optimization process, we define a refractive index profile with form

$$
n_{e q}(\rho)=\frac{A}{B+\left(\rho / \rho_{0}\right)^{C}},
$$

where $\rho_{0}$ is the source position along $\rho$. For a lens of radius $R$, with the source at $\left(\rho_{0}, \phi_{0}\right)=(R / 2,0)$ and the optimized parameters $A=3, B=2$, and $C=1.7$, we get the $n_{e q}$ profile and the ray-paths shown in Fig.1a and Fig.1b, respectively. Although the refractive index can be optimized for a different position of the source, from a practical point of view, placing several horns around an inner ring is the most convenient alternative to preserve the symmetry of the model. As represented in Fig.1b and Fig.1c, we consider this inner hole of radius $R_{\text {in }}=R / 2$.

Once the ideal distribution of $n_{e q}$ has been obtained, we proceed to its implementation by cylindrical metallic pins with variable height $[3,7]$. The MTS created by the pins is enclosed in the lower PPW. The final SW lens is presented in Fig.1c. In this work, we evaluate only the plane wave generated in the upper PPW, without introducing yet any perturbation to radiate the power. This is the reason why the cylindrical reflector has been truncated at the top layer in the simulation.

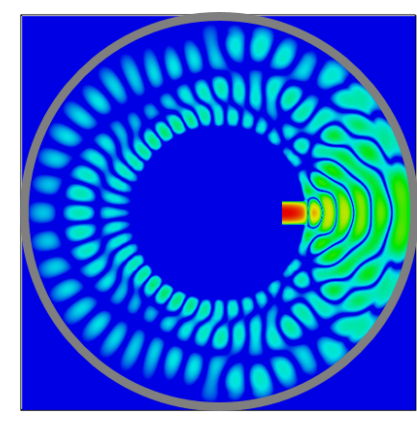

(a) Bottom layer (field amplitude)

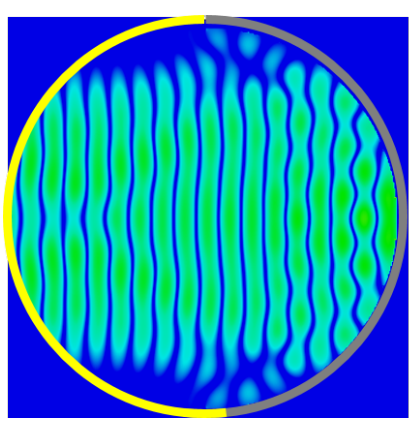

(b) Top layer (field amplitude)
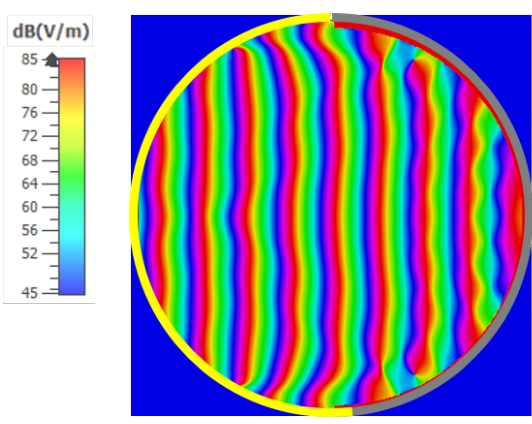

(c) Top layer (field phase)

Fig. 2: (a) Simulated field amplitude in flat lens, at lower layer. (b) Resulting plane wave in upper layer. (c) Phase of plane wave in top layer. PEC walls are represented by grey arches and absorbing BCs are in yellow.

Fig. 2 shows some preliminary numerical results at $f_{o}=30 \mathrm{GHz}$ for the field amplitude in both layers, as well as the phase in the top layer. One can observe that a plane wave is created in the top layer, after the reflection of the field from the bottom layer. This performance is maintained within the bandwidth $f=[29,31] \mathrm{GHz}$. It is 
clear that the behavior can be replicated for different positions of the horn, given the cylindrical symmetry of the system. Then, a rotation of the horn position of an angle $\phi_{0}$ will entail an equivalent variation in the plane wave angle of propagation. It is important to note that this rotation can cover the total azimuthal range (from $0^{\circ}$ to $360^{\circ}$ ), since all the design elements hold the layout symmetry. Indeed, the radial dependence of $n_{e q}$ and the cylindrical geometry of the reflector ensure the conservation of the plane wave generation when modifying $\phi_{0}$. Ordinary Luneburg lenses are usually subjected to 1-layer arrangements, with the sources on one side and the collimated beam on the other side, which limits the scanning range in azimuth to $\pm 90^{\circ}$. This constraint is overcome by the presented configuration.

\section{CONCLUSION}

We have described the design, implementation and simulation results of a Reflecting Luneburg lens. The flat MTS lens consists of two stacked PPWs and has been realized by using metallic pins with variable height on the bottom PPW. By employing this architecture, we have succeeded in creating a plane wave that propagates towards the excitation port and not beyond the rim of the lens. In this sense, the behavior is contrary to a conventional Luneburg lens. The double-layered approach along with the cylindrical reflector result in a really compact structure, where the beamformer and the radiating aperture are placed within the same horizontal area. The presented system offers an interesting alternative to classical quasi-optical beam-formers for reconfigurable antennas.

\section{ACKNOWLEDGEMENT}

This publication has been supported by the European Union through the European Regional Development Fund (ERDF), and by the French Region of Brittany, Ministry of Higher Education and Research, Rennes Métropole and Conseil Départemental 35, through the CPER Project STIC \& Ondes. It has been also partially supported by the European Space Agency under contract no. 4000127381/19/NL/AF. Finally, it has received the support of the Direction Générale de l'Armement (DGA), the École des Docteurs de l'UBL (Universit Bretagne Loire) and the Conseil Régional de Bretagne.

\section{REFERENCES}

[1] E. Martini et al., "Flat optics for surface waves," IEEE Trans. Antennas Propag., vol. 64, no. 1, pp. 155-166, Jan 2016.

[2] S. Cornbleet, "Geometrical optics reviewed: A new light on an old subject," Proc. IEEE, vol. 71, no. 4, pp. 471-502, April 1983.

[3] M. Bosiljevac, M. Casaletti, F. Caminita, Z. Sipus, and S. Maci, "Non-uniform metasurface luneburg lens antenna design," IEEE Trans. Antennas Propag., vol. 60, no. 9, pp. 4065-4073, Sep. 2012.

[4] E. Martini, M. Jr, and S. Maci, "Metasurface transformation for surface wave control," Philosophical transactions. Series A, Mathematical, physical, and engineering sciences, vol. 373, 082015.

[5] M. Huang, S. Yang, F. Gao, R. Quarfoth, and D. Sievenpiper, "A 2-D multibeam half maxwell fish-eye lens antenna using high impedance surfaces," IEEE Antennas Wireless Propag. Lett., vol. 13, pp. 365-368, 2014.

[6] M. Faenzi et al., "Metasurface antennas: New models, applications and realizations," Sci. Rep., vol. 9, p. 10178, July 2019.

[7] D. González-Ovejero, N. Chahat, R. Sauleau, G. Chattopadhyay, S. Maci, and M. Ettorre, "Additive manufactured metalonly modulated metasurface antennas," IEEE Trans. Antennas Propag., vol. 66, no. 11, pp. 6106-6114, Nov 2018.

[8] N. Chahat, B. Cook, H. Lim, and P. Estabrook, "All-metal dual-frequency rhcp high-gain antenna for a potential europa lander," IEEE Trans. Antennas Propag., vol. 66, no. 12, pp. 6791-6798, 2018.

[9] Tianxia Zhao, D. R. Jackson, J. T. Williams, H. . D. Yang, and A. A. Oliner, "2-D periodic leaky-wave antennas-part i: metal patch design," IEEE Trans. Antennas Propag., vol. 53, no. 11, pp. 3505-3514, Nov 2005.

[10] Y. B. Li et al., "Dual-physics manipulation of electromagnetic waves by system-level design of metasurfaces to reach extreme control of radiation beams," Adv. Mater. Technol., vol. 2, no. 1, p. 1600196, 2017.

[11] C. Pfeiffer and A. Grbic, "A printed, broadband luneburg lens antenna," IEEE Trans. Antennas Propag., vol. 58, no. 9, pp. 3055-3059, Sep. 2010.

[12] M. Ettorre, R. Sauleau, and L. Le Coq, "Multi-beam multi-layer leaky-wave siw pillbox antenna for millimeter-wave applications," IEEE Trans. Antennas Propag., vol. 59, no. 4, pp. 1093-1100, April 2011. 